\title{
¿Puede haber carrascales en el sur de Navarra? Una aproximación mediante la modelización de distribución de especies
}

\author{
J. Peralta ${ }^{1, *}$, N.A. Zepeda ${ }^{1}$, J.B. Imbert ${ }^{1}$ \\ (1) Departamento de Ciencias del Medio Natural, Universidad Pública de Navarra, 31006 Pamplona, Navarra, España \\ * Autor de correpondencia: J. Peralta [javier.peralta@unavarra.es]
}

> Recibido el 08 de noviembre de 2013, aceptado el 20 de diciembre de 2013

Peralta, J., Zepeda, N.A., Imbert, J.B. 2013. ¿Puede haber carrascales en el sur de Navarra? Una aproximación mediante la modelización de distribución de especies. Ecosistemas 22(3):58-65. Doi.: 10.7818/ECOS.2013.22-3.09.

De acuerdo con algunos mapas de vegetación potencial diversos factores climáticos y edáficos limitan el hábitat disponible para los bosques de carrasca (Quercus rotundifolia) en el sur de Navarra (NE de España). Este es un territorio con un intenso y antiguo uso agrícola y ganadero, lo que dificulta valorar si son las variables ambientales o factores antrópicos los que hacen que sean muy escasos los carrascales existentes. Para tratar de responder a la cuestión de si este territorio es adecuado para el desarrollo de carrascales, se construyen modelos de distribución de la carrasca con Maxent y modelos lineales generalizados (GLM), se comparan con mapas de vegetación potencial y con otros modelos generados para esta especie en el conjunto de la península ibérica. Las variables empleadas para construir los modelos son precipitación media de agosto, continentalidad, temperatura media de las máximas de enero, radiación de enero, pendiente y desarrollo del suelo; todas fueron significativas en el GLM. En Maxent la variable con mayor contribución al modelo fue la precipitación de agosto. Los distintos modelos y mapas difieren sobre todo en los límites nordeste y sur de distribución de los carrascales. Los modelos construidos en este trabajo y los ya existentes sugieren que las variables ambientales consideradas no limitan el desarrollo de carrascales, al menos en algunas zonas del tercio meridional de Navarra, no contempladas en los mapas de vegetación potencial tradicionales.

Palabras clave: Maxent; Quercus rotundifolia; cartografía de vegetación potencial; idoneidad de hábitat.

Peralta, J., Zepeda, N.A., Imbert, J.B. 2013. . 2013. Could green-oak woodlands thrive in the south of Navarre? An approximation through species distribution modelling. Ecosistemas 22(3):58-65. Doi.: 10.7818/ECOS.2013.22-3.09.

According to some potential vegetation maps, various climatic and edaphic factors limit the suitable habitat for green-oak woodlands (Quercus rotundifolia) in the South of Navarre (NE Spain). This area has been used for agriculture and livestock since ancient times, making it difficult to assess whether environmental or anthropic factors are the cause of the scarcity of green-oak woodlands. To try to answer this question green-oak distribution models were built with Maxent and generalized linear models (GLM). Their outcome was then compared with potential vegetation maps and previous models built for the Iberian Peninsula applied to data from Navarre. The environmental variables used to build the models were August mean rainfall, continentality, January mean maximum temperature, January irradiation, slope and soil development; all were significant in the GLM analysis. The variable that most contributed to Maxent model was August mean rainfall. Predictions of spatial distributions of green-oak woodlands by models and maps mainly differ for the north-eastern and southern range of Navarre. Our models and the pre-existent ones suggest that the studied environmental variables do not limit the habitat suitability for green-oak woodlands at least in some areas of southern Navarre, which were not considered in traditional potential natural vegetation maps.

Key words: Maxent; Quercus rotundifolia; potential vegetation mapping; habitat suitability.

\section{Introducción}

En diversos trabajos de geobotánica y mapas de vegetación potencial se considera que en el área central del valle del Ebro (NE de la península ibérica), de la que forma parte el sur de Navarra, no es posible el desarrollo de bosques de carrasca (Quercus rotundifolia Lam. [= Q. ilex L. subsp. ballota (Desf.) Samp.]), salvo en los cerros de mayor altitud y algunas umbrías, donde todavía se observan pequeños rodales. Las formaciones vegetales de mayor porte en este territorio serían coscojares de Quercus coccifera, lentiscares de Pistacia lentiscus y pinares de Pinus halepensis (BraunBlanquet y Bolòs 1957, Montserrat 1966, Bolòs et al. 1986, Rivas-Martínez 1987, Loidi y Báscones 2006, Peralta 1997, 2002). Los trabajos existentes hasta la fecha basan sus conclusiones acerca de la potencialidad de la vegetación en las características climáticas del territorio, la ausencia o escasa presencia de la carrasca en la zona y la existencia de otras formaciones arbustivas y arbóreas de carácter más xerófilo, consideradas climácicas.

La definición del área de distribución potencial de los carrascales tiene interés no sólo desde un punto de vista teórico. De acuerdo con la Directiva de Hábitats (Unión Europea 1992) estas formaciones son hábitat de interés y, por lo tanto, su estado de conservación debe ser objeto de una evaluación periódica. Para evaluarlo se ha de establecer un área de referencia favorable basada, entre otros factores, en el área de distribución potencial (Evans y Arvela 2011), que puede ser estimada mediante el uso de mapas de vegetación potencial tradicionales (Ihobe 2011), la elaboración de mapas de distribución histórica (NPWS 2007) o bien mediante 
modelos de distribución de especies. La disponibilidad de datos precisos de la distribución de Quercus rotundifolia en Navarra (SERA 2010) y de cartografía de diversos factores ambientales (climáticos, topográficos, edáficos) permite construir en la actualidad modelos de distribución de especies aplicando algunas de las numerosas técnicas disponibles (Franklin 2009, Miller 2010). Mediante estos modelos es posible calcular la probabilidad de presencia de la especie en distintos puntos del territorio, así como determinar los factores que contribuyen a su presencia y en qué medida (Franklin 2009, Mateo et al. 2011, Pearson 2007). A escala peninsular han sido construidos modelos para carrasca por Felicísimo et al. (2011) utlilizando el algoritmo Maxent (Phillips et al. 2006) y Ninyerola et al. (2010) con modelos lineales generalizados (GLM, de sus siglas en inglés), aunque este último está referido a Quercus ilex en sentido amplio, que incluye tanto la encina costera (Q. ilex) como la de interior (Q. rotundifolia). En el modelo de Felicísimo et al. (2011) se utilizaron sólo variables climáticas mientras que en el de Ninyerola et al. (2010) se consideraron además variables topográficas. En el valle del Ebro, ambos modelos proyectan áreas de idoneidad contrapuestas, el primero con un mapa binomial similar a los mapas de vegetación potencial tradicionales y el segundo con un mapa de probabilidad continua, con mayor idoneidad hacia la zona central de la depresión.

A escala regional, además de los factores climáticos, tienen influencia los factores topográficos, edáficos y antrópicos (Pearson y Dawson 2003). Para Navarra existe información disponible de los dos primeros factores (Ninyerola et al. 2005, Íñiguez et al. (19821992) y además se cuenta con datos de presencia de la carrasca a mayor escala (SERA 2010) que los utilizados en los modelos previos, que emplean mapas e inventarios forestales a escala 1: 50000 o inferior (Felicísimo et al. 2011, Ninyerola et al. 2010). Por ello se plantea la realización de modelos con Maxent y GLM referidos exclusivamente a la carrasca (Quercus rotundifolia), con datos de presencia más precisos e incorporando variables ambientales relevantes a escala regional, para generar mapas binomiales de presencia. Los métodos empleados en el modelado pertenecen a dos de los grandes grupos de métodos de modelación (métodos de aprendizaje automático y técnicas de regresión) y se han escogido por su amplia utilización, buen rendimiento (Elith et al. 2006, Franklin 2009) y ser los utilizados en los modelos preexistentes.

De este modo se puede comparar el resultado de los modelos anteriores, que comprenden el rango completo de distribución de la carrasca, con los realizados para Navarra incorporando variables topográficas y edáficas. En los primeros se espera que los factores de tipo climático sean los determinantes (Pearson y Dawson 2003). Esta comparación resulta útil ya que al elaborar los modelos en sólo una parte del área de distribución de la carrasca, el nicho ambiental definido se verá reducido. No obstante, en Navarra existe un fuerte gradiente ambiental, y en $160 \mathrm{~km}$ de distancia se pasa de un clima de tipo templado y lluvioso en el norte a un clima mediterráneo con rasgos de aridez en el sur (SSC 2001; Loidi y Báscones 2006); en ese gradiente se encuentra el límite septentrional de distribución de la carrasca en esta parte de Europa (Jalas y Suominen 1988), coincidente con el extremo meridional de la biorregión Eurosiberiana. El objetivo general de este trabajo es generar un mapa regional de distribución potencial de los carrascales en Navarra con un mayor nivel de detalle, considerando variables climáticas y topográficas, para resolver la inconsistencia planteada por modelos anteriores a escala peninsular. Los objetivos específicos son: (1) conocer los factores condicionantes de la distribución de la carrasca en este territorio; (2) comparar estos modelos con los previos y con mapas de vegetación potencial y (3) valorar si en el sur de Navarra es posible el desarrollo de carrascales.

\section{Material y métodos}

\section{Área de estudio}

El área de estudio comprende el conjunto de Navarra, 10400 $\mathrm{km}^{2}$, desde la biorregión Eurosiberiana del tercio norte a la Mediterránea en su porción meridional (Fig. 1). El interés, sin embargo, se centra en el sur, en la comarca geográfica de la Ribera, caracterizada por un clima mediterráneo con fuertes rasgos de aridez. La continentalidad es elevada y la precipitación media anual reducida (400 l/año), con grandes variaciones interanuales y un mínimo estival que hace que exista un largo período de sequía (SSC 2001; Loidi y Báscones 2006). Es un territorio con un intenso uso agrícola desde antaño, ocupado en un $70 \%$ por cultivos. La vegetación natural es escasa y son frecuentes formaciones arbustivas (romerales, tomillares, coscojares) y pastizales xerófilos mediterráneos (lastonares, espartales). La vegetación arbórea consiste sobre todo en pinares de Pinus halepensis y muy escasos restos de carrascal, además de las choperas de los sotos fluviales (Vicente et al. 2004).

\section{Construcción de modelos de distribución potencial}

Los modelos de distribución se construyeron con Maxent (PhiIlips et al. 2006), algoritmo basado en un modelo de máxima entropía, y con modelos lineales generalizados (GLM), utilizando una distribución de errores binomial y una función de vínculo logística (i.e. regresión logística). En ambos casos es necesario disponer de puntos de presencia de la especie y capas con las variables ambientales; además, son necesarios puntos de ausencia para los GLM y también para evaluar los modelos. Las operaciones necesarias para obtener los puntos de presencia y ausencia, relacionarlos con las capas ambientales, así como la preparación de estas últimas, se realizaron con la base de datos MS-ACCESS y los sistemas de información geográfica (SIG) DIVA-GIS (Hijmans 2012), MiraMon (Pons 2013) y Quantum GIS (Athan et al. 2012).
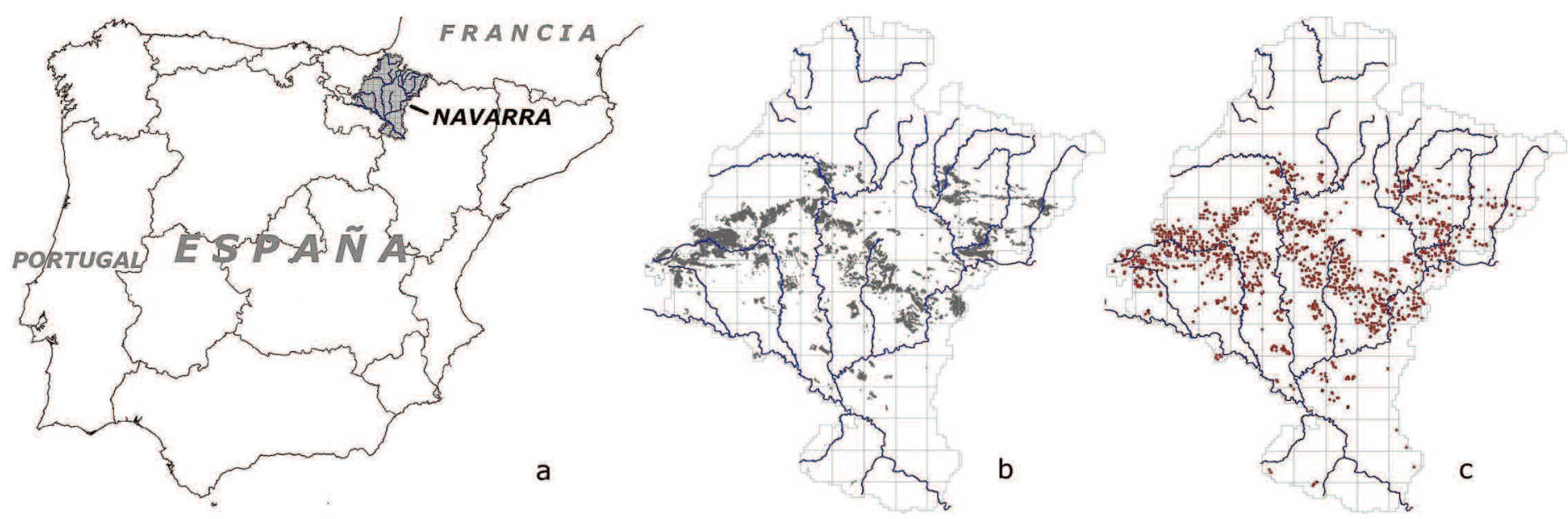

Figura 1. a) Localización del área de estudio; b) distribución de los carrascales en Navarra; c) puntos de presencia empleados en el calibrado de los modelos; en b) y c) cuadrícula UTM de $10 \mathrm{~km}^{2}$, ED50. 
Los puntos de presencia de carrascal se corresponden con lugares donde la carrasca constituye formaciones arbóreas en las que es dominante (cabida cubierta $\geq 50 \%$ ), de acuerdo con el Mapa de Ocupación del Suelo de Navarra 1:25 000 (SERA 2010; Fig. 1). Para el tercio meridional de Navarra, donde apenas sí hay recintos cartografiables de carrascal, se han obtenido datos de presencia de inventarios de vegetación, tanto de carrascales como de otras formaciones arbustivas donde la carrasca se encontraba presente (Peralta 1997, 2002). Del citado mapa se ha recogido un punto por cada kilómetro cuadrado, correspondiente al centroide del recinto con mayor superficie de carrascal en dicho cuadrado. En total se han obtenido 1767 puntos de presencia, de los que el $75 \%$ se emplearon para calibrar los modelos (Fig. 1 ) y el $25 \%$ restante para evaluarlos. De la misma forma, se obtuvieron dos conjuntos de puntos de ausencia aleatoriamente, uno para la construcción del GLM (Maxent no requiere ausencias explícitas) y otro para la evaluación de ambos modelos. De estos puntos se eliminaron los coincidentes con recintos cartográficos de carrascal, es decir, presencias, mediante un cruce con SIG (SERA 2010), quedando un total de 4268 puntos de ausencias.

Las variables ambientales inicialmente consideradas fueron 19 , de tipo climático, topográfico y edáfico (Tabla 1). Su elección se basó en el carácter limitante que pueden presentar para la carrasca y al notable gradiente que muestran en el territorio considerado. Las capas de variables climáticas se obtuvieron a partir de mapas de temperatura, radiación, precipitación (Ninyerola et al. 2005) y la combinación de algunas de éstas en índices derivados (ombrotérmico, continentalidad). El modelo digital del terreno (Ninyerola et al. 2005) proporcionó la capa de altitud a partir de la que se generaron mediante SIG las de pendiente y orientación. El desarrollo del suelo se calculó a partir del Mapa de Suelos de Navarra 1:50 000 (Íñiguez et al. 1982-1992), reclasificando mediante SIG las unidades cartográficas en una variable cualitativa ordinal con cinco categorías, indicadora del desarrollo del suelo (cuanto menos desarrollado más favorable a especies pioneras o más frugales). Todas las capas consideradas presentan una resolución de 200 x 200 m.

Los puntos de presencia de la especie se cruzaron mediante SIG con las capas de variables ambientales y se realizó un análisis de correlaciones mediante el factor de inflación de la varianza (VIF) para reducir la colinearidad. Las variables con VIF mayor que 5 fueron sucesivamente eliminadas mediante un procedimiento por pasos hacia atrás (Zuur et al.2007) realizado mediante el programa Brodgar (Zuur 2011). Finalmente quedaron seis variables ambientales para la construcción de los modelos: precipitación media de agosto, temperatura media de las máximas de enero, índice de continentalidad, radiación solar de enero, pendiente y desarrollo del suelo.

Para construir el modelo con Maxent se emplearon sólo puntos de presencia y las capas de las seis variables ambientales seleccionadas; el formato de salida empleado fue el logístico. Para el modelado con GLM se utilizaron puntos de presencia y ausencia, cada uno asociado a los valores de las variables ambientales mediante una combinación de capas con SIG. Se realizó una regresión logística con función de vínculo logit y error binomial con el programa Brodgar (Zuur 2011). Las variables se retiraron individualmente del modelo global, y se comparó el modelo sin la variable retirada con el modelo completo, aplicando pruebas de $\mathrm{F}$ para calcular la significación de cada variable; estas pruebas no son dependientes del orden de las variables (Zuur et al. 2007). Los coeficientes de las variables se emplearon para elaborar la ecuación de probabilidad de presencia. Los mapas que muestran esta probabilidad se crearon combinando mediante SIG las diferentes capas de acuerdo con dicha ecuación.

Los mapas de probabilidad obtenidos con Maxent y los generados a partir del GLM fueron cruzados con los puntos de presencia y ausencia reservados para evaluar la precisión de los modelos. De este modo se pueden calcular las clasificaciones correctas e incorrectas de dichas presencias y ausencias, y construir la matriz de confusión para distintos umbrales de probabilidad.
Tabla 1. Variables ambientales consideradas en este estudio. Se han subrayado aquellas variables que fueron seleccionadas tras el análisis de colinearidad y que fueron por tanto usadas en la construcción de los modelos.

\section{Variables ambientales}

Altitud [m]

Desarrollo del suelo [cinco categorías, de menos a más profundo]

Índice de continentalidad anual [T media mes más cálido - T media mes más frío]

Índice ombrotérmico anual [10(P/T); P: suma de precipitaciones medias mensuales; T: suma de temperaturas medias mensuales]

Índice ombrotérmico mensual: julio, agosto [10(P/T); P: precipitación media mensual; T: temperatura media mensual]

Pendiente [\%]

Precipitación media anual [l/año]

Precipitación media mensual: julio, agosto [l/mes]

Radiación solar anual $\left[\mathrm{kJ} /\left(\mathrm{m}^{2 *}\right.\right.$ día*$\left.^{*} \mu \mathrm{m}\right]$

Radiación solar mensual: enero, julio, agosto $\left[\mathrm{kJ} /\left(\mathrm{m}^{2 *} \mathrm{dí}^{*} \mu \mathrm{m}\right]\right.$

Temperatura media anual $\left[{ }^{\circ} \mathrm{C}\right]$

Temperatura media mensual de las máximas: enero, julio, agosto $\left[{ }^{\circ} \mathrm{C}\right]$

Temperatura media mensual de las mínimas: enero $\left[{ }^{\circ} \mathrm{C}\right]$

Los estadísticos empleados en la evaluación fueron el área bajo la curva ROC (AUC) y la sensibilidad, o error de omisión (Franklin 2009, Pearson 2007). La sensibilidad fue escogida frente a otros parámetros de la matriz de confusión dado que el propósito de los modelos es estimar la distribución potencial de una especie, por lo que interesa que el modelo clasifique correctamente una alta proporción de las localidades conocidas; al menos parte de las ausencias, sobre todo en el tercio sur de Navarra, pueden corresponder a hábitats apropiados no ocupados por la especie (Miller 2010, Pearson 2007). Tanto el AUC, la sensibilidad y los restantes valores derivados de la matriz de confusión (especificidad, tasa de clasificaciones correctas, Kappa de Cohen) se calcularon con el programa ROC/AUC de Schröder (2004).

Los parámetros derivados de la matriz de confusión dependen del umbral de corte que se aplique a la probabilidad continua de presencia (entre 0 y 1 ) obtenida con los modelos. Este umbral determina la presencia o ausencia de la especie a partir de dicho valor de probabilidad. El criterio empleado para definir ese punto de corte fue el de minimizar la diferencia entre sensibilidad y especificidad (Liu et al. 2005, Pearson 2007).

Para estimar si el valor de la sensibilidad difería significativamente del esperado por azar se calculó una chi-cuadrado (McDonald 2009). Para ello se transformaron los mapas de probabilidad continua obtenidos con Maxent y GLM en mapas binomiales de presencia/ausencia tras aplicar el umbral de corte elegido; así se obtuvo la superficie de presencia y ausencia de cada especie, base del cálculo de los valores esperados. El mismo procedimiento se siguió para obtener los mapas binomiales y los valores de la matriz de confusión correspondientes a los modelos realizados para la península ibérica (Felicísimo et al. 2011, Ninyerola et al. 2010) en el área correspondiente a Navarra.

Los modelos obtenidos, a los que denominaremos Maxent1 y GLM1, fueron comparados con: (1) los modelos peninsulares construidos con Maxent (Maxent2, Felicísimo et al. 2011) y GLM (GLM2, Ninyerola et al. 2010); y (2) con dos mapas de vegetación potencial a escala 1:200 000 (VP200) y 1:100 000 (VP100) (Loidi y Báscones 2006, Peralta 2011). Los modelos Maxent2, GLM2 y los mapas de vegetación se evaluaron con el mismo conjunto de puntos empleados con los restantes modelos.

\section{Resultados}

En el modelo Maxent1 la variable principal es la precipitación de agosto, con una contribución mucho mayor (58.9\%) que las demás. Superan el $10 \%$ la temperatura media de las máximas de 
enero $(17.5 \%)$ y el desarrollo del suelo (11.3\%). Las restantes variables presentan valores inferiores (Tabla 2, Fig. 2). El AUC es de 0.81 , indicando un modelo aceptable al ser superior a 0.7 (Schröder 2004); la sensibilidad es de 0.73; cuanto más se aproxima a 1 (100\% de las presencias correctamente clasificadas) indica un mejor modelo (Tabla 3). En el modelo GLM1 todas las variables ambientales son significativas (Tabla 4) así como sus coeficientes, salvo los correspondientes al desarrollo del suelo (Tabla 5). La probabilidad de presencia de la carrasca aumenta con la pendiente y disminuye cuando se incrementa el valor del índice de continentalidad, la temperatura media de máximas enero, la precipitación media de agosto o la radiación de enero. EI AUC es 0.71, también aceptable, y la sensibilidad algo menor que en el modelo Maxent1 (0.66) (Tabla 3).

En el modelo Maxent2 el AUC es de 0.68 , próximo al valor considerado indicador de un modelo aceptable, y la sensibilidad de 0.68. En el modelo con GLM2 el AUC es igual al anterior y la sensibilidad de 0.53 . Para los mapas de vegetación potencial sólo se calcularon los parámetros derivados de la matriz de confusión y no el AUC, dado el carácter binomial de estos mapas (probabilidad 0 o 1 de presencia). La sensibilidad del mapa VP100 es de 0.87 y de 0.72 en VP200. En todos los modelos y mapas de vegetación potencial el valor de la sensibilidad es significativo, lo que indica que la precisión de los modelos es mayor que la esperable por azar (Tabla 3).

En la figura 3 se muestran los mapas de presencia/ausencia construidos con los diferentes modelos, empleando los umbrales de corte indicados en la tabla 3. El porcentaje de superficie con hábitat apropiado para la carrasca de acuerdo con los modelos y los mapas de vegetación presenta una diferencia de un $23.2 \%$ entre los valores extremos. Maxent1 y VP200 son los que proyectan menores superficies (23.1\% y $24.7 \%$ respectivamente) y GLM2 el que más (46.3\%). GLM2, Maxent2, GLM1 y VP100 proyectan valores muy similares, situados en el intervalo 30.3-32.2\%.

\section{Discusión}

La contribución de las variables consideradas en los modelos para delimitar las áreas idóneas para la carrasca en el territorio estudiado es congruente con la información conocida respecto al conjunto de la península ibérica. La carrasca es un árbol planoperennifolio bien adaptado a la sequía estival característica del clima mediterráneo por sus hojas esclerófilas y su desarrollado sistema radicular (Rodà et al. 2009): la precipitación media de agosto, indicador de esta sequía, es la variable con mayor contribución en el modelo Maxent1 (Tabla 2). El signo negativo de su coeficiente en GLM1 (Tabla 5) muestra que en los territorios donde llueve más en agosto tiende a disminuir su presencia, lo que sucede hacia el norte de Navarra; cuando la sequía se reduce o desaparece, diversos bosques de caducifolios (Quercus faginea, Q. pubescens, Fagus sylvatica) y perennifolios (Pinus sylvestris) desplazan a los carrascales. Por el contrario, la mayor sequía que existe hacia el sur, podría favorecer a los bosques de Pinus halepensis y maquias de coscoja o lentisco (Braun-Blanquet y Bolòs 1957, Montserrat 1966, Rodà et al. 2009). Ambas situaciones frente al aumento y disminución de la precipitación estival quedan bien reflejadas en la respuesta de Maxent 1 (Fig. 2).

Las formaciones de carácter eurosiberiano (hayedos, robledales y pinares de Pinus sylvestris), también reemplazan a los carrascales cuando el frío invernal es prolongado o intenso y el ombroclima húmedo (Rodà et al. 2009), como sucede en el norte de Navarra. La influencia de este factor se observa en la curva de respuesta de Maxent1 frente a la temperatura media de las máximas de enero (Fig. 2). Este efecto también se produce a la inversa y por encima de cierto umbral disminuye la probabilidad de presencia, como indica el coeficiente negativo en GLM1 (Tabla 5). Esto sucede en los valles cantábricos, de clima oceánico, donde dominan bosques caducifolios, y hacia el fondo del valle del Ebro, donde como ya se ha comentado, los carrascales son muy escasos. No parece que el aumento de la temperatura media de las máximas de enero pueda tener un efecto negativo en el desarrollo de las ca-
Tabla 2. Porcentajes de contribución de las variables en el modelo Maxent1.

\begin{tabular}{lc}
\hline Variables ambientales & $\%$ \\
\hline Precipitación media de agosto & 58.9 \\
Temperatura media de máximas de enero & 17.5 \\
Desarrollo del suelo & 11.3 \\
Pendiente & 8.8 \\
Índice de continentalidad & 2.8 \\
Radiación media de enero & 0.8 \\
\hline
\end{tabular}

Tabla 3. Parámetros empleados para la evaluación de los modelos y los mapas; para la sensibilidad resultados de la prueba de chi-cuadrado.

\begin{tabular}{|c|c|c|c|c|c|c|}
\hline & Maxent1 & Maxent2 & GLM1 & GLM2 & VP100 & VP200 \\
\hline AUC & 0.81 & 0.68 & 0.71 & 0.68 & - & - \\
\hline $\begin{array}{l}\text { P umbral } \\
\text { de corte }\end{array}$ & 0.44 & 0.45 & 0.33 & 0.46 & - & - \\
\hline Sensibilidad & 0.73 & 0.68 & 0.66 & 0.53 & 0.87 & 0.72 \\
\hline$X^{2}(g \mid=1)$ & 582.7 & 239.9 & 218.5 & 74.2 & 644.8 & 504.1 \\
\hline$p x^{2}$ & $<0.001$ & $<0.001$ & $<0.001$ & $<0.001$ & $<0.001$ & $<0.001$ \\
\hline $\begin{array}{l}\text { \% superficie } \\
\text { carrasca }\end{array}$ & 23.1 & 31.6 & 32.2 & 46.3 & 30.3 & 24.7 \\
\hline Especificidad & 0.72 & 0.64 & 0.65 & 0.53 & 0.73 & 0.78 \\
\hline $\begin{array}{l}\text { Correctamente } \\
\text { clasificados }\end{array}$ & 0.73 & 0.67 & 0.64 & 0.53 & 0.77 & 0.76 \\
\hline $\begin{array}{l}\text { Kappa } \\
\text { de Cohen }\end{array}$ & 0.40 & 0.32 & 0.26 & 0.05 & 0.51 & 0.45 \\
\hline
\end{tabular}

Maxent2: Felicísimo et al. 2011; GLM2: Ninyerola et al. 2010; VP100: Peralta 2011; VP200: Loidi \& Báscones 2006

Tabla 4. Cambio en la devianza y valores de F si una variable es eliminada del modelo GLM1.

\begin{tabular}{|c|c|c|c|c|c|}
\hline Variable eliminada & gl & Devianza & AIC & $\mathbf{F}$ & $\mathbf{P}$ \\
\hline Ninguna & & 4698.4 & 4718.4 & & \\
\hline $\begin{array}{l}\text { Precipitación } \\
\text { media agosto }\end{array}$ & 1 & 5104.7 & 5122.7 & 388.0 & $<0.001$ \\
\hline Índice de continentalidad & 1 & 4788.6 & 4806.6 & 86.2 & $<0.001$ \\
\hline $\begin{array}{l}\text { Temperatura media } \\
\text { máximas enero }\end{array}$ & 1 & 4706.1 & 4724.1 & 7.4 & 0.007 \\
\hline Radiación media enero & 1 & 4731.0 & 4749.0 & 31.2 & $<0.001$ \\
\hline Pendiente & 1 & 4836.0 & 4854.0 & 131.4 & $<0.001$ \\
\hline Factor desarrollo suelo & 4 & 4849.9 & 4861.9 & 36.2 & $<0.001$ \\
\hline
\end{tabular}

Devianza del modelo nulo: 5485.4

Tabla 5. Coeficientes del modelo lineal generalizado (GLM1).

\begin{tabular}{|c|c|c|c|c|}
\hline Variables & Coeficientes & Error estándar & Test Z & $\mathbf{P}$ \\
\hline (Intercept) & 2.3120 & 0.0187 & 0.012 & 0.990 \\
\hline $\begin{array}{l}\text { Precipitación media } \\
\text { agosto }\end{array}$ & -0.0087 & 0.0005 & -17.685 & $<0.001$ \\
\hline $\begin{array}{l}\text { Índice de } \\
\text { continentalidad }\end{array}$ & -0.0597 & 0.0064 & -9.293 & $<0.001$ \\
\hline $\begin{array}{l}\text { Temperatura media } \\
\text { máximas enero }\end{array}$ & -0.0107 & 0.0039 & -2.764 & $<0.01$ \\
\hline $\begin{array}{l}\text { Radiación media } \\
\text { enero }\end{array}$ & -0.0017 & 0.0003 & -5.680 & $<0.001$ \\
\hline Pendiente & 0.0828 & 0.0073 & 11.363 & $<0.001$ \\
\hline $\begin{array}{l}\text { Factor desarrollo } \\
\text { suelo } 1\end{array}$ & 12.6500 & 0.0187 & 0.068 & 0.946 \\
\hline $\begin{array}{l}\text { Factor desarrollo } \\
\text { suelo } 2\end{array}$ & 12.2400 & 0.0187 & 0.065 & 0.948 \\
\hline $\begin{array}{l}\text { Factor desarrollo } \\
\text { suelo } 3\end{array}$ & 12.2900 & 0.0187 & 0.066 & 0.948 \\
\hline $\begin{array}{l}\text { Factor desarrollo } \\
\text { suelo } 4\end{array}$ & 11.3500 & 0.0187 & 0.061 & 0.952 \\
\hline
\end{tabular}



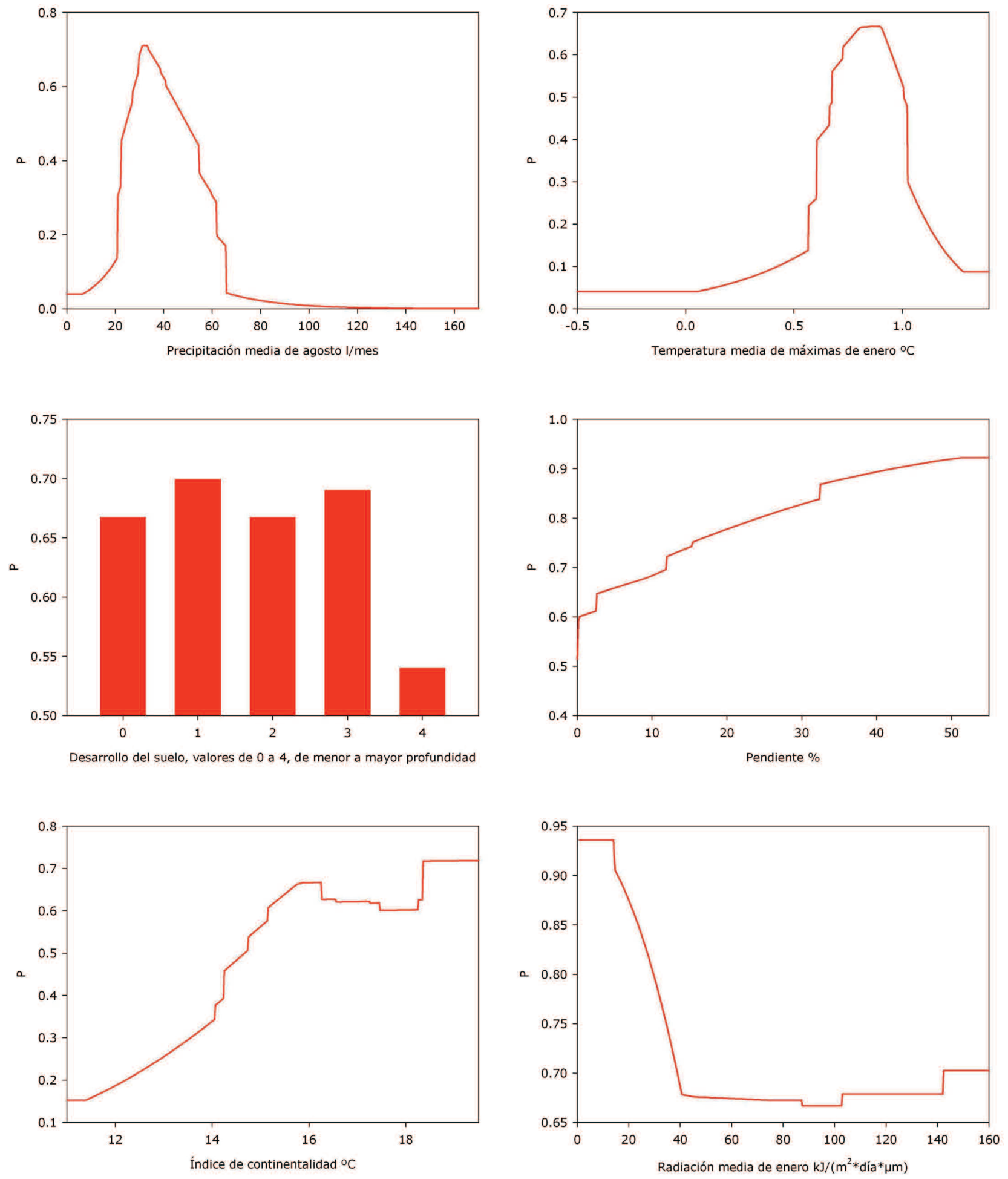

Figura 2. Curvas de respuesta de las variables para Maxent1, mostrando los cambios de la predicción logística (probabilidad de 0 a 1) en función de los valores de cada variable

rrascas, sino más bien al contrario; serían otros factores como la competencia de caducifolios en el norte u otros factores climáticos, edáficos o antrópicos en el sur, los que limitarían su presencia.

El que el aumento de la radiación mínima de enero muestre una relación negativa con la presencia de carrasca (Tabla 5, Fig. 2) podría estar relacionado con la distribución de los carrascales en distintas orientaciones de ladera; la radiación depende en gran parte de la orientación y pendiente del terreno, por lo que podría indicar que en conjunto sean más frecuentes en otras orientaciones distintas de la sur, donde la radiación es mayor.

La continentalidad presenta valores muy altos en el tercio meridional de Navarra, lo que ha constituido un argumento para justificar la ausencia de carrascales como vegetación potencial en la mayor parte de ese territorio (Bolòs et al. 1986, Loidi y Báscones 2006, Peralta 2002) a pesar de que el ombroclima, con unos 400 l/año, entra dentro del rango apropiado para estas formaciones (Rodà et al. 2009). El coeficiente negativo del índice de continentalidad en GLM1 (Tabla 5) y el decrecimiento en la respuesta en Maxent1, una vez que este índice alcanza valores altos (Fig. 2), podrían apoyar esta justificación. Sin embargo, en los valores más elevados de continentalidad aumenta esa respuesta en Maxent1, lo que está relacionado con los carrascales localizados en el nordeste y sur de Navarra, donde es máxima la continentalidad.

Finalmente, el aumento de la pendiente incrementa la probabilidad de presencia de la carrasca, como indica su coeficiente en GLM1, y disminuye en los suelos más profundos como muestra la 


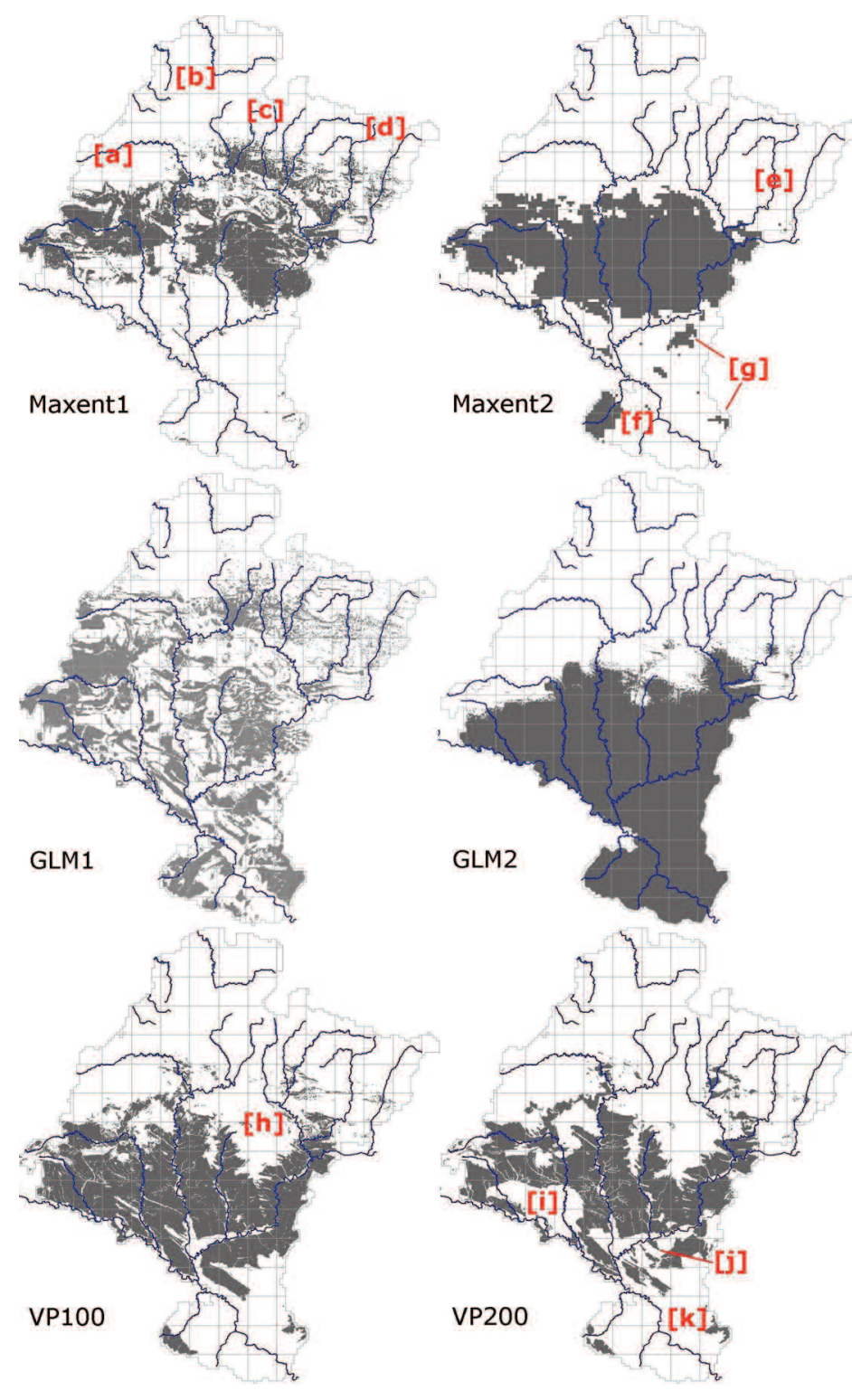

Figura 3. Mapas de presencia/ausencia generados a partir de modelos de distribución potencial de especies utilizando modelos Maxent y modelos lineales generalizados realizados en este estudio (Maxent 1, GLM1) y en estudios ya publicados (Maxent 2, Felicísimo et al. 2011; GLM2, Ninyerola et al. 2010), y mapas de vegetación ya existentes a escala 1:100 000 (VP100, Peralta 2011) y 1:200 000 (VP200, Loidi y Báscones 2006). a) sierras de Urbasa y Andia; b) valles cantábricos; c) montañas de la divisoria de aguas cantábrico-mediterránea; d) Pirineos; e) Prepirineo; f) cuenca del río Alhama; g) Bardenas; h) sierra de Ujué, cuenca de Aoiz-Lumbier; i) Ribera estellesa; j) regadíos de la acequia de Navarra; k) Valle del Ebro, Tudela.

respuesta en Maxent1 (Fig. 2). Ello concuerda con su mayor abundancia en terrenos abruptos, como sucede en otros territorios de la península ibérica, dada su eliminación desde antiguo de las zonas de relieve suave, más aptas para la agricultura (Rodà et al. 2009).

Al comparar los cuatro modelos y los dos mapas de vegetación se observa que distan de ser coincidentes (Fig. 3). En todos ellos aparece la zona central de Navarra como un hábitat idóneo para la carrasca; es lógico, dado que es donde se encuentran la mayor parte de los carrascales y por tanto la mayoría de las presencias utilizadas para calibrar los modelos y realizar los mapas (Fig. 1). También queda bien definido el límite septentrional de distribución en su parte occidental (Fig. 3a), coincidente con las sierras de Urbasa y Andia, y que marca el paso de la biorregión Mediterránea a la Eurosiberiana, salvo en GLM2 donde ese límite se dibuja algo más al sur. En este territorio los carrascales, acompañados de quejigares, dan paso a los robledales de Quercus pubescens y haye- dos. Más al norte, en el cuadrante noroeste, con ambiente más oceánico, aparecen los robledales de Q. robur, y la zona es considerada como inapropiada para los carrascales en todos los modelos (Fig. 3b), así como el resto del extremo norte donde se encuentran las montañas de la divisoria de aguas cantábrico-mediterránea (Fig. 3c) y los Pirineos (Fig. 3d).

En el cuadrante nororiental de Navarra el límite de distribución de la carrasca es discordante en los distintos modelos. En el Prepirineo, más continental, abundan los pinares de Pinus sylvestris y robledales de Quercus pubescens, acompañados por algunos hayedos en umbrías (Figura 3e). También existen algunas manchas importantes de carrascal (Fig. 1), coincidentes con algunas solanas y afloramientos rocosos con suelos someros. En los modelos Maxent1 y GLM1 aparece toda esta zona salpicada de áreas apropiadas para la especie; en el modelo GLM2 (Ninyerola et al. 2010) también se observan algunas zonas idóneas, ausentes en Maxent2 (Felicísimo et al. 2011). Estas ausencias en Maxent2 pueden deberse a que el modelo sólo incorpora variables climáticas, mientras que en este territorio en límite de área son los factores topográficos y edáficos los que parecen condicionar la presencia de carrascales. En GLM2 la presencia de algunas de estas zonas sugiere que el modelo incluye variables topográficas, pero es posible que al estar construido a escala peninsular las variables climáticas tengan mayor peso que en los modelos regionales. Los mapas de vegetación potencial reflejan la presencia de carrascales en este ámbito, aunque atribuyéndole una menor superficie que Maxent1 y GLM1. Esta diferencia puede ser debida a que la carrasca tenga un hábitat apropiado en ese territorio, aunque por la competencia de otras especies arbóreas, sobre todo Quercus pubescens y Pinus sylvestris, quede relegada a terrenos desfavorables, rocosos o con suelo somero.

A esa misma situación cabe atribuir las diferencias entre los mapas de vegetación potencial y los modelos en el centro-este de Navarra, desde la cuenca de Aoiz-Lumbier a la sierra de Ujué (Fig. $3 \mathrm{~h}$ ). En esta zona se encuentran bosques de Quercus marcescentes (Quercus faginea, Q. pubescens, Q. subpyrenaica), que serían más competitivos frente a la carrasca en los mejores terrenos, y a los que dichos mapas atribuyen la potencialidad de la vegetación.

En general ambos mapas de vegetación son muy similares. Difieren, como es lógico, en la mayor precisión del de menor escala (VP100), como se deduce de los valores de sensibilidad. También difieren en la consideración como hábitat apropiado de los afloramientos de yeso de la Ribera estellesa (Fig. 3i) y en el tratamiento que se da a zonas de regadío artificial fuera del ámbito de la vegetación riparia, no consideradas como hábitat apropiado para la carrasca por Loidi y Báscones (2006) dado su uso actual (Fig. 3j).

La distribución de los carrascales en la parte central del valle del Ebro (Figs. 3f, g, i y k) presenta grandes diferencias en los modelos y mapas de vegetación. Maxent1 es el modelo más conservador y se ajusta a las localidades conocidas, aunque deja de incorporar algunas de las presencias meridionales (Fig. $3 \mathrm{~g}$ ). Es el modelo que presenta mayor sensibilidad $(0.73)$ y menor superficie potencial (23.1\%; Tabla 3). GLM2 (Ninyerola et al. 2010) muestra una situación opuesta y proyecta como apropiado para la carrasca casi todo el territorio mediterráneo de Navarra. Presenta, sin embargo, la menor sensibilidad (0.53) de los modelos a la vez que la mayor superficie potencial (46.3\%). GLM1 es semejante a GLM2 en el sur de Navarra, aunque los terrenos llanos, muchos de ellos con suelo profundo, quedan excluidos. Maxent2 (Felicísimo et al. 2011) se asemeja más a los mapas de vegetación potencial, sobre todo a VP200, aunque amplía el territorio idóneo en el sudoeste del río Ebro, en la cuenca del río Alhama (Fig. 3f).

Como ya se ha comentado, entre las variables climáticas consideradas condicionantes de la distribución general de los carrascales se encuentran la precipitación estival, el frío invernal (Rodá et al. 2009) y la continentalidad en la Ribera de Navarra (Loidi y Báscones 2006). La precipitación ya se ha indicado que se encuentra dentro del rango apropiado para el desarrollo de los carrascales en el sur de Navarra, y que el frío invernal disminuye hacia esa zona. Por otro lado, aunque el aumento de la continentalidad, a 
partir de cierto umbral, tiende a disminuir la probabilidad de presencia de carrascales, no lo hace en sus valores más elevados.

Por lo tanto, considerando el hábitat que aparece como idóneo para los carrascales en el sur de Navarra en la mayor parte de los modelos (GLM1, GLM2, Maxent2) y que de acuerdo con estos modelos las variables climáticas, topográficas y edáficas descritas no limitan el desarrollo de los carrascales en esa zona, podemos concluir que la extensión potencial de los carrascales en este territorio es superior a la que reflejan los mapas de vegetación potencial existentes hasta la fecha (VP200, VP100) (Figs. 3f, i y k). GLM1 parece un modelo más coherente que GLM2 y Maxent2 con la distribución conocida de los carrascales, ya que define mejor el área potencial en su parte norte; además destaca respecto a GLM2 por su mayor sensibilidad ( 0.66 frente a 0.53 ) a pesar de que proyecta un área potencial inferior (32.2\% frente a $46.3 \%$ ).

Entre los factores no considerados en la construcción de los modelos se encuentran la salinidad, el alto contenido en yeso del suelo y la hidromorfía, que limitarían el desarrollo de los carrascales (Loidi y Báscones 2006, Rodá et al. 2009), pero sólo en las zonas concretas donde concurrieran dichos factores. Esta conclusión supone atribuir, indirectamente, la ausencia de los carrascales al uso del territorio, algo de lo que puede ser indicio la mayor rareza de los carrascales en zonas llanas; sin embargo, este aspecto debería ser respaldado con datos procedentes de estudios históricos (restos arqueológicos, archivos, etc.).

A la hora de valorar la utilidad de los distintos modelos hay que tener en cuenta que sólo representan los factores abióticos teóricamente apropiados para la carrasca y no incorporan factores bióticos, como la competencia de otras especies (Soberon y Peterson 2005), de ahí parte de sus diferencias con los mapas de vegetación potencial, sobre todo hacia la biorregión Eurosiberiana donde los carrascales entran en contacto con caducifolios (robledales, hayedos) y pinares de Pinus sylvestris. Esta limitación, sin embargo, no es tal en el sur de Navarra donde las formaciones arbóreas son muy reducidas y como hemos visto es difícil precisar si la ausencia de carrascales es debida a unas condiciones climáticas o edáficas inadecuadas o a una eliminación antigua de estos bosques.

Finalmente, es necesario reconocer que una limitación al modelizar la distribución potencial de bosques mediterráneos en la península ibérica es la escasez de datos de presencia por su eliminación en zonas cultivadas (Mateo et al. 2011), lo que ha de tenerse en cuenta al interpretar los modelos resultantes (Felicísimo et al. 2011). En el caso de los modelos que generamos para Navarra se ha tratado de paliar este problema con una fuente de datos de presencia de mayor resolución y con la incorporación de datos propios (Peralta 1997, 2002), si bien es posible que parte de la inconsistencia entre los distintos modelos se genere como consecuencia del grado de antropización del paisaje.

Por todo ello, para hacer un análisis más concluyente podría ser útil construir un modelado ceñido al territorio mediterráneo de este tramo del valle del Ebro, que incorpore zonas colindantes con Navarra, incluir otros factores edáficos (Dubuis et al. 2013) como la salinidad, el contenido en yesos y la hidromorfía, y ensamblar modelos realizados con distintos algoritmos para mejorar la predicción (Grenouillet et al. 2011, Marmion et al. 2009), aunque seguiría siendo un obstáculo la escasez de registros de presencia de carrasca en la zona.

\section{Referencias}

Athan, T. (coord.). 2012. Quantum GIS Development Team software. Disponible en http://www.qgis.org

Bolòs, O. de, Montserrat, P., Báscones, J.C., Creus, J. 1986. Fitogeografía. En: Floristán, A. (Dir.) Gran Atlas de Navarra vol 1: 95-102. Caja de Ahorros de Navarra. Pamplona. España

Braun-Blanquet, J., Bolòs, O. de. 1957. Les groupements végétaux du bassin moyen de l'Ebre et leur dynamisme. Anales de la estación experimental de Aula Dei 5: 1-266.

Dubuis, A., Giovanettina, S., Pellissier, L., Pottier, J., Vittoz, P., Guisan, A. 2013. Improving the prediction of plant species distribution and commu- nity composition by adding edaphic to topo-climatic variables. Journal of Vegetation Science 24: 593-606.

Elith, J., H. Graham, C., P. Anderson, R., Dudík, M., Ferrier, S., Guisan, A., et al. 2006. Novel methods improve prediction of species' distributions from occurrence data. Ecography 29: 129-151.

Evans, D., Arvela, M. 2011. Assessment and reporting under Article 17 of the Habitats Directive. Explanatory Notes and Guidelines for the period 2007-2012. European Topic Centre on Biological Diversity. Paris. France.

Felicísimo Á.M. (coord.) 2011. Impactos, vulnerabilidad y adaptación al cambio climático de la biodiversidad española. 1. Flora y vegetación. Oficina Española de Cambio Climático, Ministerio del Medio Ambiente y Medio Rural y Marino. Madrid. España. Disponible en: http://secad.unex.es/wiki/libroOECC/index.php?title=Página_Principal

Franklin, J. 2009. Mapping Species Distributions. Spatial Inference and Prediction. Cambridge University Press. Cambridge. UK.

Grenouillet, G., Buisson, L., Casajus, N. Lek, S. 2011. Ensemble modelling of species distribution: the effects of geographical and environmental ranges. Ecography 34: 9-17.

Hijmans, R.J. 2012. DIVA-GIS software for species habitat modelling, version 7.5. Disponible en: http://www.diva-gis.org/download.

Ihobe 2011. Primera evaluación del estado de conservación de los hábitats de bosque de interés comunitario en el País Vasco. Sociedad Pública del Departamento de Medio Ambiente, Planificación Territorial, Agricultura y Pesca del Gobierno Vasco. Bilbao. España. Disponible en http://www.euskadi.net/contenidos/informe_estudio/habitats_bosques/e s doc/adjuntos/09 habitat.pdf

Íñiguez, J., Sánchez-Carpintero I., Val, R., Arricibita, F. et al. 1982-1992. Mapa de Suelos de Navarra, escala 1:50 000. Universidad de Navarra. Pamplona. España.

Jalas, J., Suominen, J. (eds.) 1988. Atlas Florae Europaeae. Distribution of Vascular Plants in Europe. 2. Angiospermae (part): Salicaceae to Balanophoraceae, Polygonaceae, Chenopodiaceae to Basellaceae. Cambridge University Press, Cambridge. Gran Bretaña.

Liu, C., Berry, P.M., Dawson, T.P., Pearson, R.G. 2005. Selecting thresholds of occurrence in the prediction of species distributions. Ecography 28: 385-393.

Loidi, J., Báscones, J.C. 2006. Memoria del mapa de series de vegetación de Navarra. Gobierno de Navarra. Pamplona. Gobierno de Navarra. Pamplona. España. Disponible en http://idena.navarra.es

Marmion, M., Parviainen, M., Luoto, M., Heikkinen, R.K., Thuiller, W., 2009 Evaluation of consensus methods in predictive species distribution modelling. Diversity Distrib. 15: 59-69.

Mateo, R.G., Felicísimo, A.M., Muñoz, J. 2011. Modelos de distribución de especies: Una revisión sintética. Revista Chilena de Historia Natural 84 217-240.

McDonald, J.H. 2009. Handbook of Biological Statistics (2nd ed.). Sparky House Publishing, Baltimore, Maryland. USA. Disponible en http://udel.edu/ mcdonald/statgtestgof.html

Miller, J. 2010. Species Distribution Modelling. Geography Compass 4-6: 490-590.

Montserrat, P. 1966. La vegetación de la cuenca del Ebro. P. Cent. Pir. Biol. Exp.: 1, 1-22.

Ninyerola, M., Pons, X., Roure, J.M. 2005. Atlas climático digital de la península ibérica. Universidad Autónoma de Barcelona. Disponible en http://opengis.uab.es/wms/iberia/mms/index.htm.

Ninyerola, M., Serra-Díaz, J.M., Lloret, F. 2010. Atlas de idoneidad topoclimática de leñosas. Servidor de mapas. Universitat Autònoma de Barcelona. Disponible en http://www.opengis.uab.cat//doneitatPl/index.html

NPWS 2007. The status of EU protected habitats and species in Ireland. Vol. 3. National Parks and Wildlife Service. Government of Ireland. Dublin, Ireland. Disponible en: http://www.npws.ie/publications/euconservationstatus/NPWS 2007 Cons_Ass Backing_V3.pdf

Pearson, R.G. 2007. Species' distribution modeling for conservation educators and practitioners. Synthesis. Lessons in Conservation 3. American Museum of Natural History. New York, NY, USA. Disponible en: http://ncep.amnh.org/linc.

Pearson, R.G., Dawson, T.P. 2003. Predicting the impacts of climate change on the distribution of species: are bioclimate envelope models useful? Global Ecology and Biogeography 12: 361-371.

Peralta, J. 1997. Series de vegetación y sectorización fitoclimática de la Comarca Agraria VI. Gobierno de Navarra. Informe técnico. Pamplona. España. Disponible en: http://www.cfnavarra.es/agricultura/informacion_ agraria/MapaCultivos/htm/documentos.htm

Peralta, J. 2002. Series de vegetación y sectorización fitoclimática de la Comarca Agraria VII. Gobierno de Navarra. Informe técnico. Pamplona. 
España. Disponible en: http://www.cfnavarra.es/agricultura/informacion_agraria/MapaCultivos/htm/documentos.htm

Peralta, J. 2011. Vegetación Potencial de Navarra 1:25.000. Dpto. de Desarrollo Rural y Medio Ambiente y Administración Local, Gobierno de Navarra. Pamplona. Disponible en:

http://www.cfnavarra.es/agricultura/informacion_agraria/MapaCultivos/ $\mathrm{htm} / \mathrm{index} . \mathrm{htm}$

Philips, S.J., Anderson, R.P., Schapire, R.E. 2006. Maximum entropy modelling of species geographic distributions. Ecological Modelling 190: 231-259. Disponible en http://www.cs.princeton.edu/ schapire/maxent/

Pons, X. 2013. MiraMon software de visualización, consulta, edición y análisis de mapas de información geográfica v. 7. CREAF. Barcelona. España.

Rivas-Martínez, S. 1987. Memoria del mapa de series de vegetación de España. Ministerio de Agricultura, Pesca y Alimentación. Madrid. España.

Rodà, F., Vayreda, J., Ninyerola, M., 2009. 9340 Encinares de Quercus ilex y Quercus rotundifolia. En: VV.AA., Bases ecológicas preliminares para la conservación de los tipos de hábitat de interés comunitario en España. Ministerio de Medio Ambiente y Medio Rural y Marino. Madrid. España.

Schröder, B. 2004. ROC plotting and AUC calculation transferability test, v. 1.3-7. Institute for Geoecology. Potsdam University. Potsdam, Germany. Disponible en: http://lec.wzw.tum.de/index.php?id=67\&L=1.
SERA 2010. Mapa de Cultivos y Aprovechamientos de Navarra, escala 1:25.000. Dpto. de Desarrollo Rural y Medio Ambiente. Gobierno de Navarra. Pamplona. España. Disponible en: http://idena.navarra.es.

Soberon, J., Peterson, A.T. 2005. Interpretation of models of fundamental ecological niches and species' distributional areas. Biodiversity Informatics 2: 1-10.

SSC 2001. Estudio agroclimático de Navarra. Sección de Suelos y Climatología, Dpto. de Agricultura, Ganadería y Alimentación, Gobierno de Navarra. Pamplona. España.

Unión Europea 1992. Directiva 92/43/CEE del Consejo de 21 de mayo de 1992 relativa a la conservación de los hábitats naturales y de la fauna y flora silvestres. Diario Oficial de las Comunidades Europeas L206, 22/07/1992, pp. 7- 50.

Vicente, A.M., Donézar, M., del Barrio, F., San Roque, M. 2004. Memoria del mapa de cultivos y aprovechamientos de Navarra. Escala 1:200.000. Departamento de Agricultura Ganadería y Alimentación. Pamplona. España. http://www.cfnavarra.es/agricultura/informacion_agraria/MapaCultivos/ind ex2.html

Zuur, A.F. 2011. Brodgar software package for data exploration, univariate analysis, multivariate analysis and time series analysis 2.7.2. Highlands Statistics Ltd. Newburgh. UK.

Zuur, A.F., leno, E.N., Smith, G.M. 2007. Analyzing Ecological Data. Springer. New York. USA. 